\title{
Motion of objects embedded in lipid bilayer membranes: advection and effective viscosity
}

\author{
Brian A. Camley 1 , a) and Frank L. H. Brown ${ }^{2,3}$ \\ ${ }^{1)}$ Departments of Physics \& Astronomy and Biophysics, Johns Hopkins University, Baltimore, Maryland 21218, USA \\ ${ }^{2)}$ Department of Chemistry and Biochemistry, University of California, Santa Barbara, California 93106, USA \\ ${ }^{3)}$ Department of Physics, University of California, Santa Barbara, California 93106, USA
}

An interfacial regularized Stokeslet scheme is presented to predict the motion of solid bodies (e.g. proteins or gel-phase domains) embedded within flowing lipid bilayer membranes. The approach provides a numerical route to calculate velocities and angular velocities in complex flow fields that are not amenable to simple Faxén-like approximations. Additionally, when applied to shearing motions, the calculations yield predictions for the effective surface viscosity of dilute rigid body-laden membranes. In the case of cylindrical proteins, effective viscosity calculations are compared to two prior analytical predictions from the literature. Effective viscosity predictions for a dilute suspension of rod-shaped objects in the membrane are also presented.

\section{INTRODUCTION}

Protein motion on the surface of lipid membranes is critical to a wide variety of biological function, from cell signaling to immune respons $\mathrm{e}^{1}$. Understanding experimental trajectories of proteins and protein aggregates on membrane surfaces ${ }^{2} \mathrm{ei}-$ ther in vitro or in vivo requires theory that can predict the influence of protein shape and size on interactions with the surrounding lipid environment. The classic description of membrane protein diffusion is the Saffman-Delbrück (SD) mode ${ }^{\sqrt{3+5}}$, which describes proteins as rigid cylinders embedded in a thin, effectively two-dimensional, fluid membrane in contact with semi-infinite bulk fluids to either side - the water and cytosol. Because of this interesting combination of hydrodynamic flow in two and three dimensions, membranes are often referred to as "quasi-two-dimensional, 6 .

The SD model predicts a verifiable dependence of diffusion coefficient on object size, membrane viscosity, and the viscosities of the surrounding bulk fluids. Many experiments on protein diffusion in membranes in vitro agree with the SD model $\frac{788}{17}$, though inconsistencies have been reported by some groups ${ }^{9}$. Molecular dynamics simulations of lipid bilayers also validate the SD model, but it is essential to explicitly account for the small box sizes and periodic boundary conditions when comparing to theory in this case $\mathrm{e}^{10-14}$. In addition, the SD model and extensions to it ${ }^{15 / 16}$ have been successfully applied far beyond the original membrane context, to the dynamics of thin layers of proteins at air-water interfaces ${ }^{17}$, soap films $\sqrt{18}$, and suspended liquid crystal films $s^{1920}$, though some interesting deviations from perfect quasi-2D behavior have also been found ${ }^{21}$. Motion of larger ordered domains on the membrane surface is also described by the SD theory 22123. While deviations from SD behavior have been reported, there is no doubt that the underlying hydrodynamic picture is largely correct and should be viewed as the appropriate starting point or first-order model for studying membrane hydrodynamics. This work assumes the validity of the SD approach and extends its predictions, via numerical calculations,

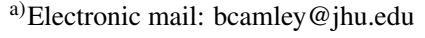

to cases where analytical predictions would be difficult or impossible.

We have previously worked on extending the SaffmanDelbrück model beyond its base assumptions, including the introduction of an interfacial regularized Stokeslet (RS) method to allow for numerical computations of diffusion coefficients and pair diffusion coefficients for membraneembedded objects of arbitrary shape 2425 . The focus of this earlier work centered around the computation of drag and mobility coefficients, i.e. the response of an object to an applied force in an otherwise quiescent membrane, which allows for prediction of diffusion coefficients via the Einstein relation. The motion of a protein or other solid body induced by flow within the membrane was not previously considered. This paper demonstrates how to generalize the interfacial RS approach to compute the dynamics of force- and torque-free objects embedded in an external flow field. Additionally, it is shown that these computations can be used to predict the effective surface viscosity of a rigid-body-laden membrane at low concentration, i.e. the intrinsic viscosity of a membrane-embedded object or the "membrane Einstein correction". Carrying out this calculation numerically, as opposed to analytically $\sqrt[626]{ }$, opens up the possibility to consider arbitrarily shaped inclusions and not only simple cylindrical bodies. In particular, this work shows that linear oligomers display an increased intrinsic viscosity relative to their monomeric counterparts, potentially allowing a characterization of membrane protein oligomerization state through determination of membrane viscosity.

\section{REGULARIZED STOKESLETS FOR FORCE- AND TORQUE-FREE EMBEDDED OBJECTS}

As for traditional three-dimensional geometries, the quasitwo-dimensional Stokes equations appropriate for the SD geometry can be solved by a Green's function approach. Within the membrane,

$$
v_{i}(\mathbf{r})=\int d \mathbf{r}^{\prime} T_{i j}^{S D}\left(\mathbf{r}-\mathbf{r}^{\prime}\right) f_{j}\left(\mathbf{r}^{\prime}\right)
$$

where $v_{i}$ are velocity components in the plane of the membrane, $T_{i j}^{S D}(\mathbf{r})$ is the membrane Oseen tensor (Green's func- 
Background flow

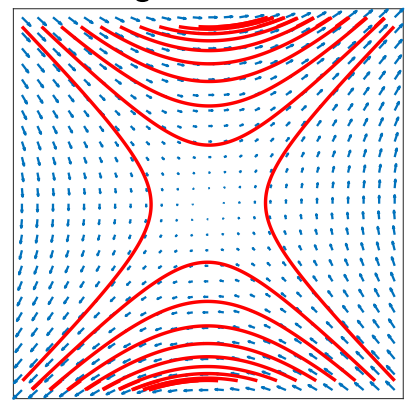

Flow with inclusion

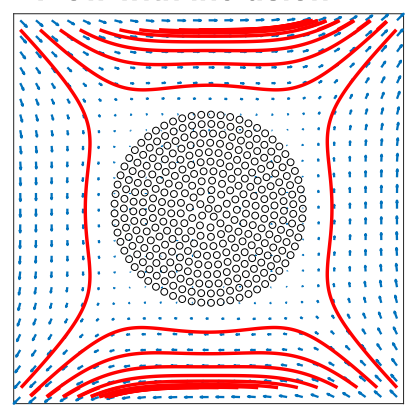

FIG. 1. Effect of an embedded force- and torque-free circular object of radius $a$ on background flow $\mathbf{v}^{\text {amb }}=(y, x)$. Flows are shown by vector field and streamline. The object is represented by $N=331$ blobs, with spacing $s=0.1 a(\epsilon=0.05 a)$, and $L_{s d}=100 a$; the object radius is $a=1$. This is a coarser representation than that used for most calculations in this paper. To the precision in the RS method $\left(\sim 10^{-4}\right)$, the object remains stationary under the flow.

tion for velocity response to point forcing), and $f_{j}$ the force density in the fluid. (Here, and throughout the paper, the Einstein summation convention is used. $i$ and $j$ run over the $x, y$ Cartesian dimensions spanning the membrane surface.) $T_{i j}^{S D}$ in Eq. 1 is most conveniently specified by its Fourier transform 2728 ,

$$
T_{i j}^{S D}(\mathbf{k})=\frac{1}{\eta_{m} k^{2}+2 \eta_{f} k}\left(\delta_{i j}-\frac{k_{i} k_{j}}{k^{2}}\right)
$$

where $\eta_{m}$ is the membrane surface viscosity and $\eta_{f}$ the viscosity of the bulk fluid exterior to the membrane. (Our convention for Fourier transforms is $f(\mathbf{k})=\int d^{2} r e^{-i \mathbf{k} \cdot \mathbf{r}} f(\mathbf{r})$, $f(\mathbf{r})=\int \frac{d^{2} k}{(2 \pi)^{2}} e^{i \mathbf{k} \cdot \mathbf{r}} f(\mathbf{k})$. ) This Oseen tensor reduces to that of a two-dimensional fluid for lengths well below the characteristic SD length $L_{s d} \equiv \eta_{m} / 2 \eta_{f}$, and becomes similar to that of a three-dimensional fluid for lengths beyond $L_{s d}$ (Ref. 6).

Eq. 1 predicts the velocity $\mathbf{v}(\mathbf{r})$ of a homogeneous membrane devoid of any particles as generated by external forcing elsewhere in the fluid $\mathbf{f}\left(\mathbf{r}^{\prime}\right)$. This formulation is not obviously useful for the description of solid bodies within the membrane. However, the method of regularized Stokeslets (RS) $\sqrt{2930}$ uses the homogeneous Green's function formulation to provide numerical solutions for hydrodynamic problems that include embedded solid particles. The essential trick is to recognize that, within creeping flow, there is no mathematical distinction between a solid body coupled to the surrounding fluid via no-slip boundaries and a "fluid" region occupying the same space as the solid body, but constrained to undergo only rigid-body motion. Thus, solid bodies within the RS approach are represented as localized fluid regions subject to forcing, but constrained to translate and rotate as a solid body. (The regularized Stokeslet approach is closely related to earlier "shell" methods in three dimensions ${ }^{31 / 33}$. In the interfacial context, related methods have been suggested by Levine, Liverpool, and Mackintosh ${ }^{1516}$ and extended by other ${ }^{34 \mid 35 !}$.)

In practice, the interfacial RS scheme works as follows. (See Refs. 24 and 25 for further details.) The continuous force distribution $\mathbf{f}(\mathbf{r})$ in Eq. 1 is replaced with a discrete collection of "blobs" with profile $\phi_{\epsilon}(\mathbf{r})=\frac{1}{2 \pi \epsilon^{2}} e^{-r^{2} / 2 \epsilon^{2}}$. (See Fig. 1.) A blob centered at $\mathbf{r}^{\prime}$, has a force distribution $\mathbf{f}(\mathbf{r})=\mathbf{g} \phi_{\epsilon}\left(\mathbf{r}-\mathbf{r}^{\prime}\right)$ and creates the velocity response $v_{i}(\mathbf{r})=T_{i j}^{S D}\left(\mathbf{r}-\mathbf{r}^{\prime} ; \epsilon\right) g_{j}$. Numerically practical formulas for $T_{i j}^{S D}\left(\mathbf{r}-\mathbf{r}^{\prime} ; \epsilon\right)=\int d \mathbf{r}^{\prime \prime} T_{i j}^{S D}\left(\mathbf{r}-\mathbf{r}^{\prime \prime}\right) \phi_{\epsilon}\left(\mathbf{r}^{\prime \prime}-\mathbf{r}^{\prime}\right)$ and implementation details can be found in $24 \mid 25$.

A membrane-embedded solid object is discretized into $N$ blobs centered at locations $\mathbf{R}_{n}$. External forces exerted on blob $n$ are denoted $g\left[\mathbf{R}_{n}\right]$ and the velocity response to these forces at a point $\mathbf{r}$ is then $v_{i}(\mathbf{r})=\sum_{n} T_{i j}^{S D}\left(\mathbf{r}-\mathbf{R}_{n} ; \epsilon\right) g_{j}\left[\mathbf{R}_{n}\right]$. Because the Stokes equations are linear, if there is a preexisting ambient lipid flow with velocity $\mathbf{v}^{\mathrm{amb}}(\mathbf{r})$, the membrane flow at each blob becomes:

$$
v_{i}\left[\mathbf{R}_{m}\right]=v_{i}^{\mathrm{amb}}\left(\mathbf{R}_{m}\right)+\sum_{n} T_{i j}^{S D}\left(\mathbf{R}_{m}-\mathbf{R}_{n} ; \epsilon\right) g_{j}\left[\mathbf{R}_{n}\right]
$$

the second contribution indicating the influence of any external forcing on the blobs. It is worth emphasizing that "external forcing" includes the forces of constraint within the object that act to maintain rigid body motion in defiance of $\mathbf{v}^{\mathrm{amb}}$. These forces are imposed upon the homogeneous fluid and share the same sign convention as would a force from an external field acting on the fluid.

To determine the motion of a solid body or particle carried under the influence of $\mathbf{v}^{\mathrm{amb}}(\mathbf{r})$, but absent any additional external forcing, requires the determination of $g_{j}$ s that 1) create only rigid body motion within the body, i.e.

$$
\mathbf{v}\left[\mathbf{R}_{m}\right]=\mathbf{U}+\boldsymbol{\Omega} \times \mathbf{R}_{m}
$$

with $\mathbf{U}$ and $\boldsymbol{\Omega}$ specifying the particle's velocity and angular velocity; 2) combine to yield vanishing total external force and torque on the particle, i.e.

$$
\sum_{n} \mathbf{g}\left[\mathbf{R}_{n}\right]=0, \quad \sum_{n} \mathbf{R}_{\mathbf{n}} \times \mathbf{g}\left[\mathbf{R}_{n}\right]=0
$$

(assuming the centroid of the blobs is at the origin); and 3) satisfy Eq. 3 Eqs. 3 - 5 represent $4 N+3$ equations in $4 N+3$ unknowns and could, in principle, be naively solved to yield $\mathbf{v}\left[\mathbf{R}_{m}\right]$ (2N scalars), $\mathbf{g}\left[\mathbf{R}_{n}\right]$ (2N scalars), $\mathbf{U}$ (2 scalars) and $\boldsymbol{\Omega}$ (1 scalar). In practice, it is convenient to equate Eqs. 3 and 4 by removing the blob velocities as independent variables. The remaining $2 N+3$ equations in $2 N+3$ unknowns can be solved to yield $\mathbf{g}\left[\mathbf{R}_{n}\right], \mathbf{U}$ and $\boldsymbol{\Omega}$. Then, $\mathbf{v}(\mathbf{r})$ for any point $\mathbf{r}$ may be directly computed from Eq. 4 or Eq. 3 It is convenient to explicitly use the linearity of Eq. $\overline{3}$ to simplify this solution.

First, a set of forces $g_{j}\left[\mathbf{R}_{n}\right]$ is determined from the superposition of four individual problems:

$$
\begin{aligned}
-v_{i}^{\mathrm{amb}}\left(\mathbf{R}_{m}\right) & =\sum_{n} T_{i j}^{S D}\left(\mathbf{R}_{m}-\mathbf{R}_{n} ; \epsilon\right) g_{j}^{\text {cancel }}\left[\mathbf{R}_{n}\right] \\
\hat{\mathbf{x}} & =\sum_{n} T_{i j}^{S D}\left(\mathbf{R}_{m}-\mathbf{R}_{n} ; \epsilon\right) g_{j}^{\mathrm{x} \text {-trans }}\left[\mathbf{R}_{n}\right] \\
\hat{\mathbf{y}} & =\sum_{n} T_{i j}^{S D}\left(\mathbf{R}_{m}-\mathbf{R}_{n} ; \epsilon\right) g_{j}^{\mathrm{y} \text {-trans }}\left[\mathbf{R}_{n}\right] \\
\hat{\mathbf{z}} \times \mathbf{R}_{m} & =\sum_{n} T_{i j}^{S D}\left(\mathbf{R}_{m}-\mathbf{R}_{n} ; \epsilon\right) g_{j}^{\mathrm{rot}}\left[\mathbf{R}_{n}\right]
\end{aligned}
$$


Here, $\mathbf{g}^{\text {cancel }}$ are the forces required to cancel the background flow, resulting in an object that is not moving at all, $\mathrm{g}^{\mathrm{x} \text {-trans }}$ and $\mathrm{g}^{\mathrm{y} \text {-trans }}$ generate uniform $x$ and $y$ translation with unit velocity, and $\mathrm{g}^{\text {rot }}$ creates rotation with unit angular velocity. The blob forces for each individual problem are deter- mined via the GMRES method, as in Ref. 24. By combining Eqs. 6 - 9 and comparing with Eq. 3, it is clear that $\mathbf{g}=\mathbf{g}^{\text {cancel }}+U_{x} \mathbf{g}^{\mathrm{x} \text {-trans }}+U_{y} \mathbf{g}^{\mathrm{y} \text {-trans }}+\Omega \mathbf{g}^{\text {rot }}$ creates a flow with $v\left[\mathbf{R}_{m}\right]=\mathbf{U}+\boldsymbol{\Omega} \times \mathbf{R}_{m}$, i.e. perfect rigid body motion. Requiring total force and torque to vanish, (Eq. 5) yields

$$
\left(\begin{array}{ccc}
\sum_{n} g_{x}^{\mathrm{x}-\text { trans }} & \sum_{n} g_{x}^{\mathrm{y} \text {-trans }} & \sum_{n} g_{x}^{\mathrm{rot}} \\
\sum_{n} g_{y}^{\mathrm{x} \text {-trans }} & \sum_{n} g_{y}^{\mathrm{y}-\text { trans }} & \sum_{n} g_{y}^{\text {rot }} \\
\sum_{n} \mathbf{R}_{n} \times \mathbf{g}^{\mathrm{x} \text {-trans }} & \sum_{n} \mathbf{R}_{n} \times \mathbf{g}^{\mathrm{y} \text {-trans }} & \sum_{n} \mathbf{R}_{n} \times \mathbf{g}^{\mathrm{rot}}
\end{array}\right)\left(\begin{array}{c}
U_{x} \\
U_{y} \\
\Omega
\end{array}\right)=\left(\begin{array}{c}
-\sum_{n} g_{x}^{\text {cancel }}\left[\mathbf{R}_{n}\right] \\
-\sum_{n} g_{y}^{\text {cancel }}\left[\mathbf{R}_{n}\right] \\
-\sum_{n}\left(\mathbf{R}_{n} \times \mathbf{g}^{\text {cancel }}\left[\mathbf{R}_{n}\right]\right)_{z}
\end{array}\right)
$$

This $3 \times 3$ linear system can easily be solved in order to find the values of $\Omega$ and $U$ required to make the object force-free. (Eq. 10 may also be easily generalized in order to specify constraints on total force and torque, if it is necessary to consider a particle with an applied force acting on it.)

As a simple test case, consider a force- and torque-free cylinder localized at the origin in a simple extensional flow $\mathbf{v}^{\mathrm{amb}}=(y, x)$. By symmetry, the velocity and angular velocity of the particle are expected to be zero. This test case is displayed in Fig. 1 , as expected $U_{x}, U_{y}$, and $\Omega$ are small (all with absolute value $<10^{-4}$, with signs that can change depending on the spacing and specific details of the blobs chosen.) Throughout this paper, unitless velocities will generally be reported; because of the linearity of the Stokes equations, the overall scale of the velocity is only important in setting the absolute value of the force and torque. The flow in Fig. 1 results from a relatively rough discretization (only 331 blobs and $\epsilon=0.05 a$, where $a$ is the particle radius). For detailed predictions, extrapolation to infinite blobs and zero spacing must be carried out 2431,33 . In this work, final extrapolated results are calculated by fixing $\epsilon$ to half of the characteristic spacing of the discretization while reducing the spacing between blobs as specified in particular examples.

\section{ADVECTION OF MEMBRANE-EMBEDDED OBJECTS: BEYOND THE SIMPLEST FAXÉN RELATIONSHIPS}

If a force-free sphere of radius $a$ is embedded in a three-dimensional ambient flow field $\mathbf{v}^{\text {amb }}$ at position $\mathbf{r}$, its velocity is given by the Faxén relationship, $\mathbf{U}=$ $\mathbf{v}^{\mathrm{amb}}(\mathbf{r})+\frac{1}{6} a^{2} \nabla^{2} \mathbf{v}^{\mathrm{amb}}(\mathbf{r})$, with a similar result for the angular velocity 36 . Because of the finite size of the sphere, it does not track exactly the imposed flow at its center of mass, but rather includes an average of the flow over its spatial envelope. For membranes, Oppenheimer and Diamant derived approximate Faxén relationships ${ }^{6}$; for force- and torque-free cylindrical particles, these are

$$
\begin{aligned}
& \mathbf{U} \approx\left[1+\frac{1}{4} a^{2} \nabla^{2}\right] \mathbf{v}^{\mathrm{amb}}(\mathbf{r}) \\
& \mathbf{\Omega} \approx \frac{1}{2}\left[1+\frac{1}{8} a^{2} \nabla^{2}\right] \nabla \times \mathbf{v}^{\mathrm{amb}}(\mathbf{r})
\end{aligned}
$$

where the approximation indicates that these results only hold in the limit of $a \ll L_{s d}$, and could have higher-order corrections proportional to $a^{4} \nabla^{4} \mathbf{v}^{\mathrm{amb}}$.

Eqs. 11 and 12 are valid in the appropriate limit, but the interfacial RS method can capture interesting systematic deviations from these predictions. In Fig. 2, results for a cylindrical object in an arbitrarily chosen background field $\mathbf{v}^{\mathrm{amb}}$ are presented. When $L_{s d}$ is decreased, so that the assumptions of Oppenheimer and Diamant no longer hold, there can be deviations from Eqs. 11 12 , these effects are generally small when the first term of Eq. 11 is large (as in Fig. 23), but can be relatively large $(\sim 30 \%)$ when the leading order term is small, e.g. if the mean velocity at the object center of mass is small (Fig. 2C). Corrections to the angular velocity appear to be very small (Fig. 2D). There is a small $(<0.2 \%)$ deviation from the analytical results of Ref. 6 in Fig. $2 \mathrm{D}$, even in the appropriate limit. The effect is exaggerated on the plot due to the scale of the $\Omega$ axis; a numerical error of $0.2 \%$ is entirely consistent with the accuracy of the interfacial RS approach 24 . However, this error can be large in a relative sense as the error does not become smaller when the predicted angular velocity approaches zero. It is important to be aware of the limitations of the numerical methodology - we do not expect the $\mathrm{RS}$ method to produce values that are accurate to below $10^{-3}$ of the typical velocity scale without significantly more refinement than we perform here.

The results of Fig. 2 show the predicted velocity of a membrane-embedded object at a single point in space and time, demonstrating some deviations from Eq. 11. It is also possible to integrate the motion of an object through time, predicting the particle's trajectory when embedded in a stationary external lipid flow. Doing so can reveal these deviations more dramatically. (This is a purely hydrodynamic calculation, neglecting thermal motion.) In Fig. 3, motion is tracked by computing the velocity of an object embedded in a lipid flow, and then evolving the position of the center of mass of that object by $\mathbf{r}_{\mathrm{cm}}(t+\Delta t)=\mathbf{r}_{\mathrm{cm}}(t)+\mathbf{U} \Delta t$. The velocity $\mathbf{U}$ is then updated for the object's new location and the procedure is repeated and iterated. The resulting trajectories are shown in Fig. 3, similar trajectories can be computed for comparison purposes directly from Eq. 11. In the limit of a smooth flow field and $a \ll L_{s d}$, the regularized Stokeslet method and Eq. 11 agree well (Fig. 3A). However, for a more rapidly varying background flow field, the simple Faxén relationship pre- 

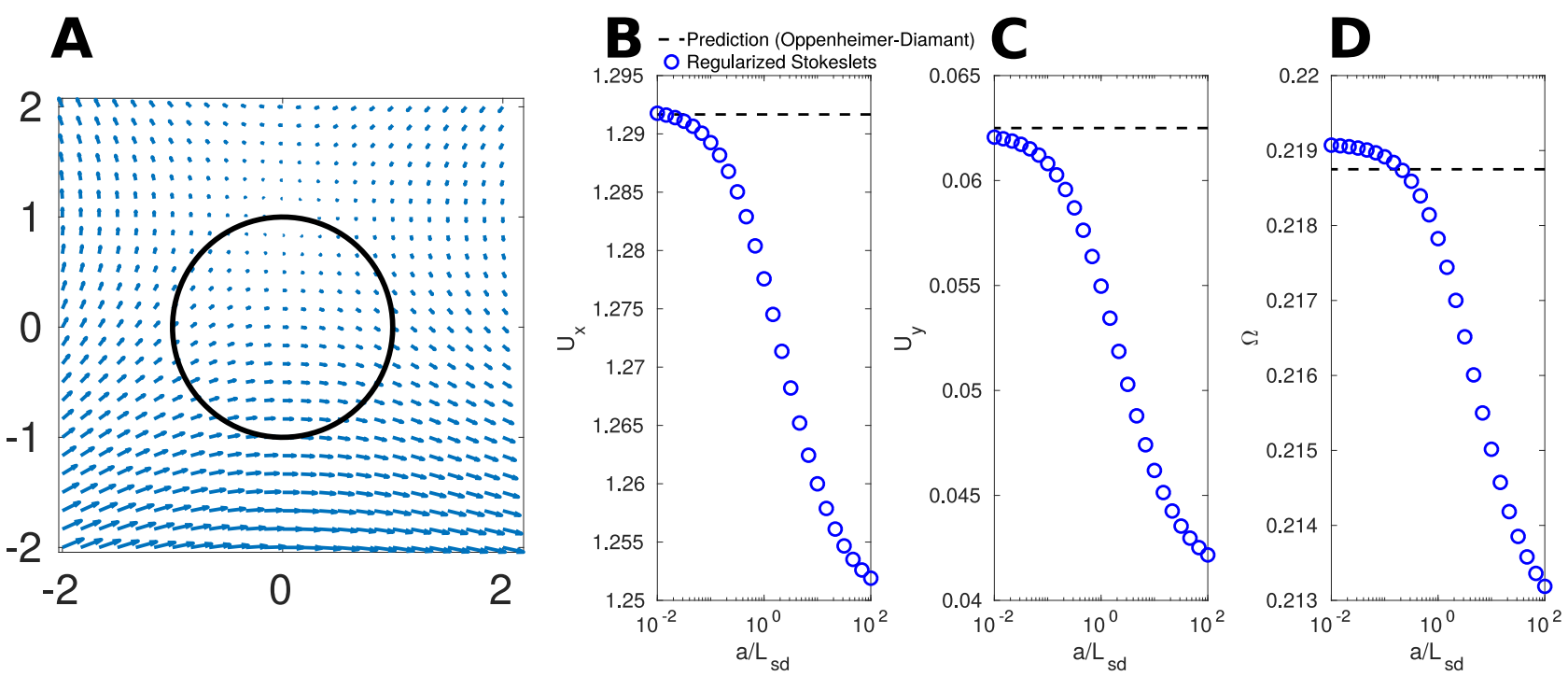

FIG. 2. Computation of object motion in external flow field $\mathbf{v}^{\text {amb }}=\left(-(y-1)+\frac{1}{8}(y-1)^{2}-\frac{1}{24}(y-1)^{3},-x+\frac{1}{8} x^{2}+\frac{1}{24} x^{3}\right)$. In this calculation, the size of the object is kept fixed (radius $a=1$ ) but the Saffman-Delbrück length is varied. A: Illustration of flow field and location and size of object; only the background flow field is shown, not the effect of the embedded object. B: $U_{x}$ (unitless) does not strongly deviate from the prediction of Eq. 11 , this occurs because the dominating term is that $U_{x} \approx v_{x}^{\mathrm{amb}}(0)$. C: Because $v_{y}^{\mathrm{amb}}(0)=0, U_{y}$ depends strongly on the local change in $\mathbf{v}^{\text {amb; }}$; this term shows a slightly stronger dependence on the Saffman-Delbrück length. D: Prediction of angular velocity. Even in the limit of $a \ll L_{s d}$, a small $(<0.2 \%)$ error remains. Relatively weak deviations occur for $a \gg L_{s d}$. Results are extrapolated from a range of spacings, $s=0.05 a, 0.1 a, 0.15 a, 0.2 a$ with $\epsilon=s / 2$.

dicts a trajectory that is significantly more oscillatory than the one found by the regularized Stokeslet method (Fig. $3 \mathrm{~B}$ ). This occurs because the Faxén relationship has neglected higherorder derivatives, and when the background flow field develops structure on the scale of $a$, the truncation is invalid. Although it is not immediately apparent in Fig. $3 \mathrm{~B}$, the wiggles in the Faxén trajectory do result from the Laplacian term, as is clear from Fig. 3 CD.

In both Fig. 2 and Fig. 3, comparing between the Faxén relationships and the interfacial RS method, it is important to choose flow fields with a sufficient number of nonzero derivatives. For instance, $\mathbf{v}^{\mathrm{amb}}=(-y, x)$ would misleadingly give the impression that there are no significant deviations from Eq. 11. because $\nabla^{2} \mathbf{v}^{\mathrm{amb}}=0$, and the particle is simply advected along the streamlines.

\section{COMPUTING EFFECTIVE VISCOSITY}

In Ref. 6, Oppenheimer and Diamant describe how to determine the effective viscosity of a dilute solid-body-laden membrane. Their approach is similar in spirit to the calculations underlying Eqs. 11 and 12 and is subject to the same assumptions, namely that the solid bodies are cylindrical and that the object radius is much smaller than $L_{s d}$. The interfacial RS approach may be used to relax these assumptions, extending the original Oppenheimer Diamant calculation to determine the the effective viscosity for a dilute suspension of arbitrarily shaped and sized bodies within the membrane.

Following Ref. 6, the basic idea for this calculation is to subject the membrane to a steady point force at the origin that generates an ambient flow field determined by $T^{S D}(\mathbf{r})$, and observe how the presence of an ensemble of solid bodies within the lipid bilayer distorts the average long-distance response of the membrane away from the ambient flow. If a rigid body is embedded in a membrane with a background flow $\mathbf{v}^{\mathrm{amb}}(\mathbf{r})$, the body will follow the flow and rotate in response to the local velocity and circulation, but the rigid particle can not deform in response to a local shear. The fact that the particle cannot deform means that there will be a net force dipole (and higher order multipoles as well) on the particle and a compensating force dipole (of opposite sign) imparted by the particle back into the membrane. Because of the linearity of the Stokes equations, this force dipole must be linearly proportional to the background velocity. If the background flow is sufficiently smooth the force dipole must be proportional to the appropriate gradients of the flow in the absence of the object ${ }^{6}$,

$$
S_{i j} \approx-\alpha \eta_{m} A_{p}\left(\partial_{i} v_{j}^{\mathrm{amb}}+\partial_{j} v_{i}^{\mathrm{amb}}\right)
$$

where the force dipole $S_{i j}=\frac{1}{2} \int d^{2} r\left[r_{i} f_{j}(\mathbf{r})+r_{j} f_{i}(\mathbf{r})\right]$, and $\alpha$ is a unitless number. Dimensional analysis indicates that $\alpha$ depends only on the object shape and $\sqrt{A_{p}} / L_{S D}$, where $A_{p}$ is the area of the object. (Note the sign difference in Eq.13 from Ref. 6. The convention here is that $S_{i j}$ is the force dipole imparted by the particle on the membrane in response to the hydrodynamic dipole, $S_{h y}=-S$, acting on the particle.) This relation assumes that the background flow is slowly-varying on the length scale $a$ of the embedded object, so higher-order terms, e.g. $a^{2} \nabla^{2} \mathbf{v}^{\mathrm{amb}}$, can be neglected. Ref. 6 finds $\alpha=2$ for a circular object with radius $a \ll L_{s d}$. Eq. 13 will only hold for an isotropic object. If the object is anisotropic, Eq.13 

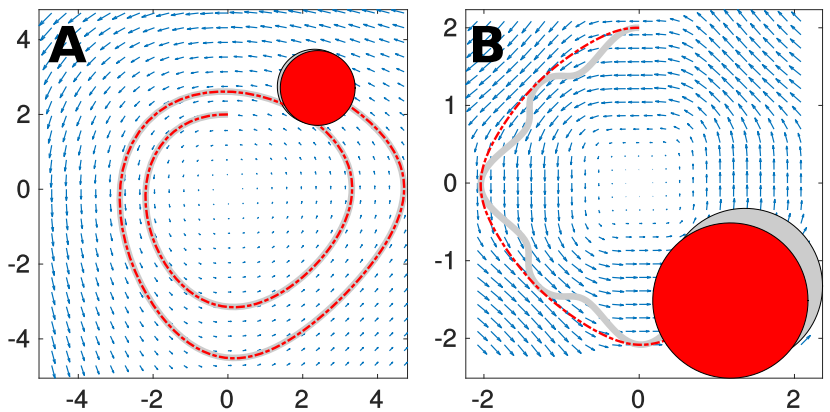

Laplacian of velocity

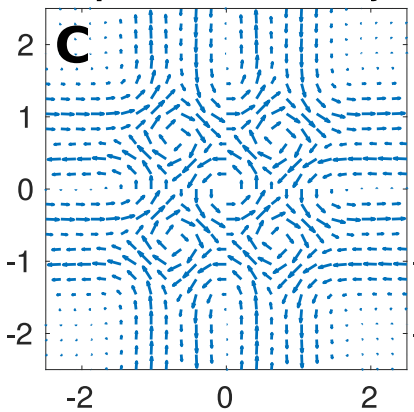

Predicted by Faxen

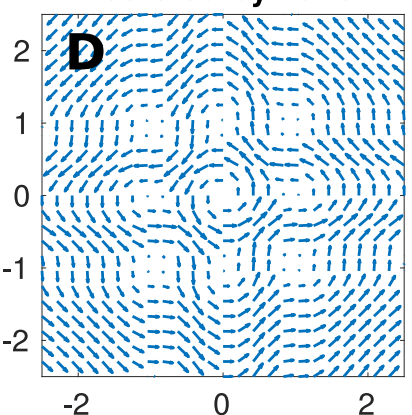

FIG. 3. In both $\mathbf{A}$ and $\mathbf{B}$, the motion of a membrane-embedded object in a background flow is computed by the approximate Faxén relationship, Eq. 11](thick gray line) and by the interfacial RS approach (red dash-dot line). The gray and red circles indicate the final position of the objects given by the Faxén relationship and RS, respectively. In both panels, $L_{s d}=100 a$, and the axis labels are in units of the object radius $a$, and with blob spacing $s=0.1 a$ and $\epsilon=0.05 a$ fixed. The background flow fields are $\mathbf{v}^{\mathrm{amb}}=\left(-y-\frac{1}{8} y^{2}, x-\frac{1}{8} x^{2}\right)$ (A) and $\mathbf{v}^{\mathrm{amb}}=\left(-\frac{y^{3}}{1+y^{4}}, \frac{x^{3}}{1+x^{4}}\right)(\mathbf{B})$. Time step used is $\Delta t=0.1$. Panels $\mathbf{C}$ and $\mathbf{D}$ help explain why the Faxén approximation fails for the flow field of Panel $\mathbf{B}$. C shows the Laplacian of $\mathbf{v}^{\mathrm{amb}}$ for Panel $\mathbf{B}$, while $\mathbf{D}$ shows the Faxén prediction's flow field, $\left[1+\frac{a^{2}}{4} \nabla^{2}\right] \mathbf{v}^{\text {amb }}$, leading to the unphysical oscillatory trajectory displayed in panel $\mathbf{B}$.

is expected to hold only after averaging over particle orientation.

The value of $\alpha$ in Eq. 13 determines the effective viscosity of a membrane with dilutely embedded objects. In the Appendix, following Ref. 6, it is shown that a membrane with area fraction $\phi \ll 1$ of solid inclusions responds, on average, as if it were a pure membrane with effective viscosity

$$
\eta_{m}^{\text {eff }}=\eta_{m}(1+\alpha \phi)
$$

In other words, $\alpha=\lim _{\phi \rightarrow 0} \frac{\eta_{m}^{\text {eff }}-\eta_{m}}{\eta_{m} \phi}$ is the "intrinsic membrane viscosity" for the embedded objects within the membrane. Eq. 14 is valid and $\alpha$ is meaningful only in the dilute limit of small $\phi$. Simulations suggest that Eq. 14 may be reasonable up to $\phi \approx 0.1$.

To compute $\alpha$ for cylindrical particles, interfacial RS calculations are performed. The particle is placed in a background flow $\mathbf{v}^{\mathrm{amb}}(\mathbf{r})=(x,-y)$ and the force-free $g_{i}\left[\mathbf{R}_{n}\right]$ 's are determined as in Sec. II $\mathbf{v}^{\mathrm{amb}}(\mathbf{r})=(x,-y)$ is a convenient form to choose, because it involves a pure shearing motion and it is impossible for higher-derivative terms to complicate Eq. 13 The stresslet can then be computed as $S_{i j}=\frac{1}{2} \sum_{n}\left(R_{n, i} g_{j}\left[\mathbf{R}_{n}\right]+R_{n, j} g_{i}\left[\mathbf{R}_{n}\right]\right)$ and compared to the predictions of Eq. 13 . Given the choice of ambient flow, $S_{x x}=-S_{y y}=-2 \alpha \eta_{m} A_{p}$ and $S_{x y}=S_{y x}=0$ and $\alpha$ follows immediately. In practice, $S_{i j}$ is determined at many different resolutions, and the results are linearly interpolated to estimate $S_{i j}$ as $\epsilon \rightarrow 0$. These extrapolated results are presented in Fig. 4 .

Consistent with the asymptotic prediction of Oppenheimer and Diamant, the numerical predictions return $\alpha \rightarrow 2$ as $a \ll L_{s d}$ (Fig. 4). This contrasts with the result of Henle and Levine ${ }^{26}$, who, using a different definition of effective viscosity predict that $\alpha \rightarrow 3$ as $a \ll L_{s d}$ (Fig. 4). The present work employs a definition of effective viscosity identical to that of Oppenheimer and Diamant, so it may not be surprising to find agreement with their results. Beyond the limit $a \ll L_{s d}$ treated analytically by Ref. 6, $\alpha$ increases, as predicted qualitatively by Henle and Levine ${ }^{26}$. In fact, simply multiplying Henle and Levine's results by a factor of $2 / 3$, to force agreement at small $a / L_{s d}$, yields excellent agreement over the full range of $L_{s d}$ values (Fig. 4). The origin of the factor of $2 / 3$ is not understood.

The linear growth of $\alpha$ at large values of $a / L_{S D}$ (see Fig. 4) reflects the fact that solid objects embedded within the membrane influence the hydrodynamics of the membrane system, even when the bare membrane viscosity becomes vanishingly small; their presence affects the flow of the surrounding wate ${ }^{526}$. This point was also noted in the original work by Henle and Levine ${ }^{26}$. For the effect of the objects to remain finite in this limit, $\alpha$ must increase linearly in $a / L_{s d}$, to overcome the explicit factors of $\eta_{m}$ in Eqs. 13 and 14. Alternatively, when $a \gg L_{S D}$, it is possible to formulate the influence of membrane-embedded particles in terms of an effective bulk viscosity for the fluid surrounding the membrane ${ }^{26}$; the bulk intrinsic viscosity in this formulation saturates to a constant when $a \gg L_{S D}$. The unbounded growth of $\alpha$ in Fig. 4 is not the indication of a physical divergence, but simply reflects the traditional conventions used in Eqs. 13 and 14 , where the leading effect of rigid inclusions is expressed as being proportional to the product $\eta_{m} \cdot \alpha$.

The interfacial RS approach can also determine the intrinsic viscosity of arbitrarily-shaped objects, if one additional step is added to the numerics. As noted above, Eq. 13 should only be expected to hold for isotropic particles. Indeed, when $S_{i j}$ for an anisotropic object is calculated, Eq. 13 can be violated. For example, under the flow $\mathbf{v}^{\mathrm{amb}}=(x,-y)$, it is no longer the case that $S_{x x}=-S_{y y}$. However, if $S_{i j}$ is determined for different orientations $\theta$ of an anisotropic object, and these orientations are averaged over as $\bar{S}_{i j}=\frac{1}{2 \pi} \int_{0}^{2 \pi} d \theta S_{i j}(\theta)$, then Eq. 13 holds for $\bar{S}_{i j}$. Appendix A shows that it is this orientationally-averaged stresslet that reports the intrinsic viscosity, assuming object orientations are uniformly distributed. This averaging increases the cost of calculation, as individual RS calculations must be performed at each orientation. (We present an alternate approach which can compute this averaging analytically, significantly reducing computational cost, in Appendix B.)

To summarize, the algorithm for computation of effective 


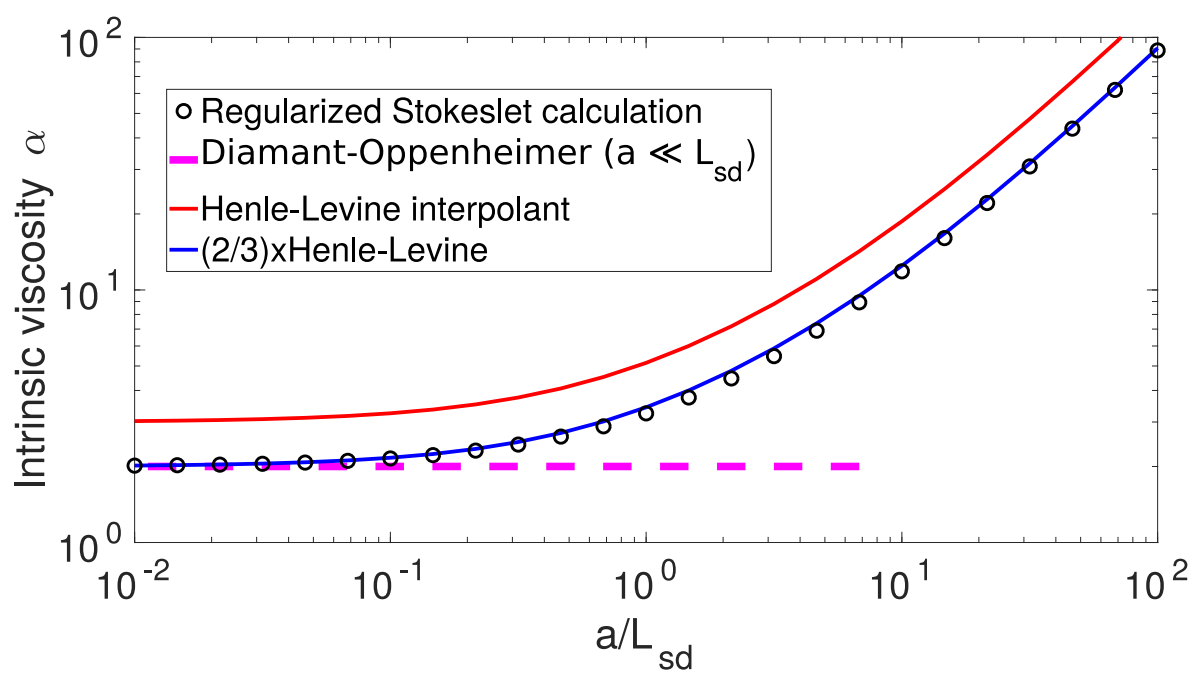

FIG. 4. Comparison between numerical RS results and literature predictions for suspensions of cylindrical particles (with radius $a$ ) in a membrane. The numerical results match Ref. 6 in the appropriate limit of $a \ll L_{s d}$, but disagree with those of Ref. 26, which defines the effective viscosity differently. However, simply rescaling the results of Ref. 26 by a factor of $2 / 3$ gives good agreement over all $L_{s d}$ values (a maximum disagreement of about $7 \%$ ). The solid lines are calculated using the interpolating function 26 : $\alpha \approx f\left(a / L_{s d}\right)$, with $f(z)=12 \pi^{-2} \ln (1+z)+\pi^{-2}(1+z)^{-1}\left[3 \pi^{2}+\left(3 \pi^{2}+8 \pi-12\right) z+4 \pi z^{2}\right]$. It is possible that the disagreement between RS numerics and the rescaled full predictions of Ref. 26 may be significantly less than $7 \%$, as Ref. 26 reports full numerical results that fall slightly below the interpolant curve, similar to what is seen for the RS numerics. Reported RS results are extrapolated from a range of spacings $s=0.05 a, 0.1 a, 0.15 a, \cdots 0.4 a$ with $\epsilon=s / 2$.

viscosity is:

1. Set up a discretization for particle shape with a particular spacing $s$ and regularization scale $\epsilon=s / 2$, with the particle centroid at the origin.

2. Solve for the rigid-body response of the particle in the external flow field $\mathbf{v}^{\mathrm{amb}}(\mathbf{r})=(x,-y)$ as in Section [II keeping a constraint of zero net force and zero net torque on the object. This solution provides a set of forces exerted on the blobs representing the object, $\mathrm{g}\left[\mathbf{R}_{n}\right]$.

3. Given these forces, compute the stresslet $S_{i j}(\epsilon)=$ $\frac{1}{2} \sum_{n}\left(R_{n, i} g_{j}\left[\mathbf{R}_{n}\right]+R_{n, j} g_{i}\left[\mathbf{R}_{n}\right]\right)$.

4. Repeat steps 1.3. after having rotated the particle around the origin. Average over many rotations to approximate $\bar{S}_{i j}(s)=\frac{1}{2 \pi} \int d \theta S_{i j}(s, \theta)$. (This step can be skipped for rotationally symmetric objects).

5. Repeat steps 114 for increasingly refined spacings $s$ (keeping $\epsilon=s / 2$ ), and then use linear extrapolation to estimate $\bar{S}_{i j}(s \rightarrow 0)$.

6. The intrinsic viscosity $\alpha$ is given by $\alpha=\bar{S}_{y y}(s \rightarrow$ $0) \times\left[2 A_{p} \eta_{m}\right]^{-1}$.

As a further application of this approach, the intrinsic viscosity of a suspension of rigid linear oligomers of circular monomers has been calculated. The intrinsic viscosity increases as a function of the length of the chain (Fig. 5). Stated differently, the effective viscosity of the membrane is expected to increase when the oligomerization state is increased while maintaining a fixed area fraction of embedded objects; the linear assemblies have a larger effect on viscosity than if they are broken apart. Similar results for the intrinsic viscosity are found for chains of three-dimensional spheres in bulk fluid ${ }^{32}$. Other papers have also addressed the hydrodynamic drag on extended bodies in membranes ${ }^{15 \mid 16 / 38}$, but not the intrinsic viscosity arising from them.

It is worth remarking that intrinsic viscosities are always defined in the limit of small area fractions $\phi \rightarrow 0$. The present work makes no attempt to quantify the range of $\phi$ values over which the present calculations may be reliable. In particular, it could be the case that longer oligomers show deviations from theoretical predictions earlier (i.e. at smaller $\phi$ values) than shorter oligomers. Previous work suggests that the linear correction works surprisingly well even to sizable area fractions for compact particles ${ }^{37}$, but possible steric or hydrodynamic interactions may become more relevant for elongated particles, and it is not obvious whether the linear correction to viscosity would be preserved for such high area fractions.

The calculations presented in this section closely parallel the analytical approach of Oppenheimer and Diamant ${ }^{6}$. It is also possible to compute the membrane effective viscosity via the grand resistance matrix formalism 3 for solid bodies within the membrane. This rephrasing of the problem more closely resembles the mobility/diffusion calculations in Refs. 24 and 25 and is presented in Appendix B. One advantage of phrasing our central problem in terms of resistance tensors is that the orientational averaging may be carried out analytically, leading to an order-of-magnitude speedup in computation. 


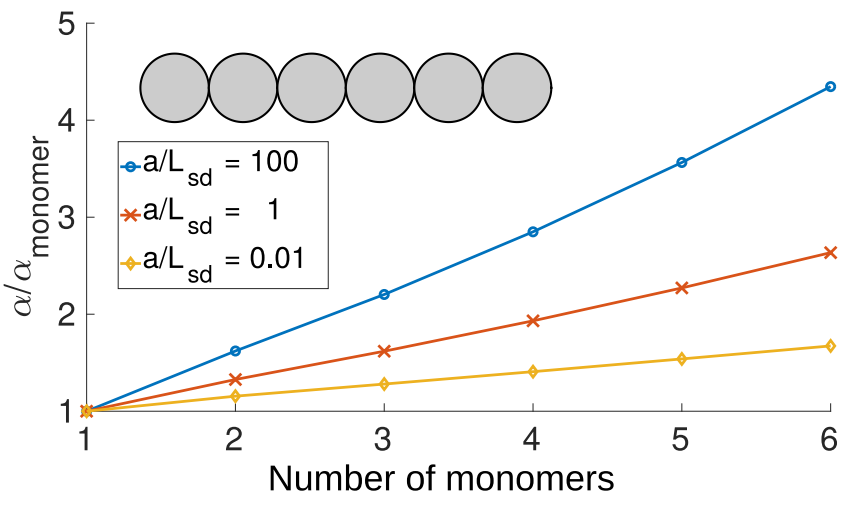

FIG. 5. Intrinsic viscosity of rigid linear oligomers of circular objects of radius $a . \alpha$ is plotted as a function of the number of monomers in the oligomer. $\alpha$ increases as the number of monomers increases, and increases more quickly (relative to its original value) for objects where $a / L_{s d}$ is larger. This simulation is computed from extrapolation over spacings $s=0.05 a, 0.08 a, 0.11 a, 0.14 a$ and averaging over ten equally spaced rotations.

\section{DISCUSSION AND CONCLUSIONS}

This paper extends the interfacial regularized Stokeslet scheme $e^{24 / 25}$ to allow simulating the motion of torque- and force-free objects advected and rotated by an ambient flow field. These calculations reproduce, in the appropriate limits, approximate Faxén relationships developed in Ref. 6, and also capture deviations from approximate predictions when particles are larger than the Saffman-Delbrück length or when the background flow is highly variable. The present approach also allows for a full calculation of the effective viscosity of a rigid-body-laden membrane in the limit of low concentration, i.e. the membrane Einstein correction. Consistent with Ref. 6. cylindrical solid inclusions of radius much smaller than the Saffman-Delbrück length yield an effective membrane viscosity of $\eta_{m}^{\text {eff }} \approx \eta_{m}(1+2 \phi)$, where $\phi$ is the area fraction of the inclusions. The effective viscosity predictions of Ref. 26 have also been confirmed, albeit with the introduction of a rescaling factor of $2 / 3$; this same factor appears in comparing Ref. 6 to the asymptotic (small particle) limit of Ref. 26 and was previously attributed to the different definitions of effective viscosity in these two works. This work shows that this systematic difference is preserved beyond the limit of small particle sizes. The numerical tools used to confirm these earlier works are immediately transferable to compute effective viscosities of non-circular objects. For example, rigid linear membrane protein chains have increased intrinsic viscosity relative to monomeric membrane proteins, suggesting that membrane protein configurations and oligomerization state could potentially be extracted from measurements of membrane viscosity. The results here provide the numerical tools required to make this possible. However, further study will be required to determine whether experimental measurements of membrane viscosity ${ }^{39}$.44 can be made sufficiently precise to gain useful information from this approach.

The present results may also prove useful in interpreting molecular dynamics simulations of lipid bilayers. Recent molecular simulations have found that increasing the concentration of proteins in the bilayer does increase the effective viscosity of the membrane, but were unable to discriminate between the Oppenheimer-Diamant and Henle-Levine predictions ${ }^{45}$. Fig. 5 suggests that studying oligomerized proteins may improve the accuracy of these measurements, as the effect of oligomers on membrane viscosity should be larger.

Various authors have used molecular simulations to study the dynamics of membranes crowded with proteins ${ }^{46-50}$. Extending the present intrinsic viscosity calculations to high protein area fractions is nontrivial. Effective viscosity calculations in bulk fluids become complicated outside the dilute limit, due to the competition between hydrodynamic, Brownian, steric, and adhesive effects ${ }^{36 \mid 51-53\}}$, with phenomenological models commonly used ${ }^{54}$. However, the present study highlights a clear physical effect in the dilute regime that should be observable in these detailed simulations and experiments. This may allow for an increasingly quantitative interpretation of dilute systems in terms of effective viscosities. A first step toward understanding complicated crowded membranes may be a careful comparison of theory/numerics, simulations and experiments in dilute membrane systems, where we have a better quantitative understanding of the models.

\section{ACKNOWLEDGMENTS}

This work was supported in part by the National Science Foundation (grant No. CHE-1800352). We thank Haim Diamant and Naomi Oppenheimer for helpful discussions and comments on this work.

\section{CODE AVAILABILITY}

Code to reproduce all of the figures shown here is available at https://github.com/bcamley/ Effective-viscosity-reproduce

\section{Appendix A: Deriving effective viscosity}

The arguments of Ref. 6 are repeated here and slightly generalized to justify the numerical determination of effective viscosity via interfacial RS calculations.

Suppose a point force, $\mathbf{f}$, is applied to a homogeneous membrane at the origin, leading to a flow field $v_{i}^{(0)}(\mathbf{r})=$ $T_{i j}^{S D}(\mathbf{r}) f_{j}$, with $T_{i j}^{S D}$ the Oseen tensor given by Eq. 2 Now, if the membrane is not homogeneous, but rather includes a rigid object embedded in the membrane at position $\mathbf{r}^{\prime}$, that object is subject to a hydrodynamic force dipole, which is compensated by the force dipole by the object acting on the membrane: $S_{i j}$. When averaged over all possible object orientations, this stresslet is (Eq. 13)

$$
\bar{S}_{i j}\left(\mathbf{r}^{\prime}\right)=-\alpha \eta_{m} A_{p}\left[\partial_{i} T_{j k}^{S D}\left(\mathbf{r}^{\prime}\right)+\partial_{j} T_{i k}^{S D}\left(\mathbf{r}^{\prime}\right)\right] f_{k}
$$


with $\alpha$ determined in practice via the interfacial RS method described in Sec. IV $S$ alters the homogeneous flow from $\mathbf{v}^{(0)}(\mathbf{r})$ to $\mathbf{v}^{(0)}(\mathbf{r})+\delta \mathbf{v}(\mathbf{r})$, with $\delta v_{i}=-S_{k j}\left(\mathbf{r}^{\prime}\right) \partial_{k} T_{i j}^{S D}(\mathbf{r}-$ $\mathbf{r}^{\prime}$ ) (this follows from the multipole expansion of the force distribution ${ }^{36}$ ). Averaging over the contributions of $\mathcal{N}$ randomly oriented identical objects, randomly scattered throughout a membrane of area $A_{m e m}$, yields

$$
\begin{aligned}
\left\langle\delta v_{i}(\mathbf{r})\right\rangle & =-\frac{\mathcal{N}}{A_{m e m}} \int_{A_{m e m}} d^{2} r^{\prime} \bar{S}_{k j}\left(\mathbf{r}^{\prime}\right) \partial_{k} T_{i j}^{S D}\left(\mathbf{r}-\mathbf{r}^{\prime}\right) \\
& =-\frac{\phi}{A_{p}} \int_{A_{m e m}} d^{2} r^{\prime} \bar{S}_{k j}\left(\mathbf{r}^{\prime}\right) \partial_{k} T_{i j}^{S D}\left(\mathbf{r}-\mathbf{r}^{\prime}\right) .(\mathrm{A} 2)
\end{aligned}
$$

$\phi=\mathcal{N} A_{p} / A_{\text {mem }}$ is the area fraction of particles with $A_{p}$ the area of each particle. It should be clear that the above derivation assumes the absence of particle-particle correlations, neglects the contributions of higher order force multipoles and considers only single-particle corrections to $\delta \mathbf{v}(\mathbf{r})$. Though these approximations are imperfect in general, they are expected to be valid in the small $\phi$ limit assumed in this work. It is also assumed that the applied force is not exerted for a long enough time for the particles to rearrange in response to the applied flow; previous simulations indicate this is not a significant issue, so long as $\mathbf{f}$ remains in the linear response regime. 37 .

Fourier transforming Eq. A2 yields

$$
\left\langle\delta v_{i}(\mathbf{q})\right\rangle=-\frac{\phi}{A_{p}} \bar{S}_{k j}(\mathbf{q}) i q_{k} T_{i j}^{S D}(\mathbf{q})
$$

and Eq. A1 and Eq. 2 imply (note that $q_{i} T_{i j}^{S D}(\mathbf{q})=0$ )

$$
i q_{k} \bar{S}_{k j}(\mathbf{q})=\alpha \eta_{m} A_{p} q^{2} T_{j m}^{S D}(\mathbf{q}) f_{m}
$$

Therefore,

$$
\begin{aligned}
\left\langle\delta v_{i}(\mathbf{q})\right\rangle & =-\phi \alpha \eta_{m} q^{2} T_{i j}^{S D}(\mathbf{q}) T_{j m}^{S D}(\mathbf{q}) f_{m} \\
& =-\alpha \phi \frac{q}{q+L_{s d}^{-1}} T_{i m}^{S D}(\mathbf{q}) f_{m} .
\end{aligned}
$$

The velocity response due to point forcing at the origin and including the effect of an ensemble of randomly distributed particles is thus

$$
\begin{aligned}
v_{i}(\mathbf{q}) & =v_{i}^{(0)}(\mathbf{q})+\left\langle\delta v_{i}(\mathbf{q})\right\rangle \equiv T_{i j}^{\mathrm{eff}}(\mathbf{q}) f_{j} \\
T_{i j}^{\mathrm{eff}}(\mathbf{q}) & =\left(1-\alpha \phi \frac{q}{q+L_{s d}^{-1}}\right) T_{i j}^{S D}(\mathbf{q}) \\
& \approx \frac{1}{\eta_{m}^{\mathrm{eff}} q^{2}+2 \eta_{f} q}\left(\delta_{i j}-\frac{q_{i} q_{j}}{q^{2}}\right) .
\end{aligned}
$$

The final line expresses that fact that, to linear order in $\phi$, $T_{i j}^{\text {eff }}=\left.T_{i j}^{S D}\right|_{\eta_{m}=\eta_{m}^{\text {eff }}}$, with $\eta_{m}^{\text {eff }}=\eta_{m}(1+\alpha \phi)$.

\section{Appendix B: Grand Resistance Matrix Formulation}

In earlier work 24 , the interfacial RS approach was used to compute the forces and torques exerted on rigid bodies embedded in a membrane. In particular, the force $\mathbf{F}$ and torque $\tau$ required to push a particle with velocity $\mathbf{U}$ and angular velocity $\Omega$ were determined. The related drag/mobility coefficients were then used to determine translational and rotational diffusion coefficients. Because of the linearity of the Stokes equations, the forces and torques must be linear in the velocities,

$$
\left(\begin{array}{c}
\mathbf{F}_{h y} \\
\tau_{h y}
\end{array}\right)=-R\left(\begin{array}{c}
\mathbf{U} \\
\Omega
\end{array}\right),
$$

where $R$ is the $3 \times 3$ "resistance matrix". Here, $\mathbf{F}_{h y}=-\mathbf{F}$ and $\tau_{h y}=-\tau$ are the hydrodynamic force and torque resisting the external forcing. The hydrodynamic and external forces (torques) must exactly compensate one another under the steady-state conditions of creeping flow.

A similar linear relationship can be developed for objects embedded in an external flow $\mathbf{v}^{\mathrm{amb}}$, creating the "grand resistance matrix' $\sqrt{36}, \mathcal{R}$. If the external flow is slowly-varying enough on the scale of the particle, it can be treated as linear, and broken down as $\mathbf{v}^{\mathrm{amb}}(\mathbf{r}) \approx \mathbf{U}^{\infty}+\Omega^{\infty} \times \mathbf{r}+E^{\infty} \cdot \mathbf{r}$, where the linear flow has been broken into a rotational term and a straining flow.

While a rigid object can rotate or translate in response to an externally imposed flow, it cannot deform. In resisting an attempted deformation by the surrounding fluid, it will exert forces on the fluid; at lowest order in the multipole expansion, this generates a net force dipole with strength $S_{i j}=-S_{i j}^{h y}$ exactly compensating the hydrodynamic dipole imposed by the external flow on the object. The force, torque, and force dipole exerted on an object will thus be given by a linear relationship of the form 36

$$
\begin{aligned}
\left(\begin{array}{c}
\mathbf{F}_{h y} \\
\tau_{h y} \\
S_{h y}
\end{array}\right) & =\mathcal{R}\left(\begin{array}{c}
\mathbf{U}^{\infty}-\mathbf{U} \\
\Omega^{\infty}-\Omega \\
E^{\infty}
\end{array}\right) \\
& =\left(\begin{array}{ccc}
A & \widetilde{B} & \widetilde{G} \\
B & C & \widetilde{H} \\
G & H & M
\end{array}\right)\left(\begin{array}{c}
\mathbf{U}^{\infty}-\mathbf{U} \\
\Omega^{\infty}-\Omega \\
E^{\infty}
\end{array}\right)
\end{aligned}
$$

Eq. B2 introduces a simplifying notation: blocks of the grand resistance matrix are scalars (e.g. $C$ ), vectors (e.g. $B$ ), and higher-order tensors (e.g. $A$ or $M$ ). Multiplication implies contraction across the indices, i.e. Eq. B2 states that if $\mathbf{U}=$ $\mathbf{U}^{\infty}$ and $\Omega=\Omega^{\infty}, S=M E^{\infty}$, which is to be taken as $S_{i j}=M_{i j k l} E_{k l}^{\infty}$. In addition, there are important symmetry relationships in these terms that follow from the Reciprocal Theorem 36 . In the quasi-two-dimensional system under study, where the only nonzero components of the angular velocity and torque are in the $z$ direction, these relationships are:

$$
\begin{aligned}
A_{i j} & =A_{j i} \\
B_{i} & =\widetilde{B}_{i} \\
G_{i j k} & =\widetilde{G}_{k i j} \\
H_{i j} & =\widetilde{H}_{i j} \\
M_{i j k l} & =M_{k l i j}
\end{aligned}
$$

where there is no symmetry relationship for $C$ because it is a scalar. The grand resistance matrix can be used to simply 
compute the effective viscosity of a rigid body-laden membrane, as will be shown below.

The grand resistance matrix $\mathcal{R}$ can be calculated as a straightforward extension of the results of Ref. 24. The basic approach is to choose the velocity of the blob points to be consistent with a rigid body motion, find the forces $g_{j}\left[\mathbf{R}_{n}\right]$ that generate these velocities when subjected to $\mathbf{v}^{\mathrm{amb}}(\mathbf{r})=$ $\mathbf{U}^{\infty}+\Omega^{\infty} \times \mathbf{r}+E^{\infty} \cdot \mathbf{r}$, and then use the blob forces to compute $\mathbf{F}, \tau$, and $S_{i j}$, which allow one to read off the matrix elements of Eq. B2 For instance, to find the (translational) drag coefficient matrix $A_{i j}$, start with Eq. 3 and choose $v_{i}\left(R_{m}\right)=U_{i}$ and $\mathbf{v}^{\mathrm{amb}}=0$. The blob forces $\mathbf{g}\left[\mathbf{R}_{n}\right]$ that generate this motion are determined numerically and are used to compute the total force on the particle $\mathbf{F}=\sum_{n} \mathbf{g}\left[\mathbf{R}_{n}\right]$ that drives the translational motion. The hydrodynamic drag force $\mathbf{F}_{h y}=-\mathbf{F}$. Then, from Eq. B2 read off $\mathbf{F}_{h y}=$ $-\sum_{n} \mathbf{g}\left[\mathbf{R}_{n}\right]=A\left(\mathbf{U}^{\infty}-\mathbf{U}\right)=-A \mathbf{U}$. For the choice $\mathbf{U}=\hat{\mathbf{x}}, A_{x x}=\sum_{n} g_{x}\left[\mathbf{R}_{n}\right]$ and $A_{y x}=\sum_{n} g_{y}\left[\mathbf{R}_{n}\right]$. Choosing $\mathbf{U}=\hat{\mathbf{y}}$ allows the reconstruction of the remaining components of $A$. This process can be extended straightforwardly for nearly all of the components of $\mathcal{R}$. Furthermore, the symmetry relationships of Eq. B3 obviate the need to perform all the calculations that one might naively expect were needed.

There is one subtlety in constructing $M_{i j k l}$. To access $M_{i j k l}$, one would like to choose $\mathbf{U}=0, \Omega=0$, and $v^{\text {amb }}=-E_{i j} r_{j}$. For this choice, Eq. B2 suggests that $S_{i j}^{h y}=-S_{i j}=-\frac{1}{2} \sum_{n}\left(R_{n, i} g_{j}\left[\mathbf{R}_{n}\right]+R_{n, j} g_{i}\left[\mathbf{R}_{n}\right]\right)=$ $-M_{i j k l} E_{k l}$. In principle, then, it should be possible to choose $E_{k l}=1$ for one component $k l$, e.g. $k l=x y$, and zero otherwise. Then it would be possible to determine $M_{i j x y}=$ $\frac{1}{2} \sum_{n}\left(R_{n, i} g_{j}\left[\mathbf{R}_{n}\right]+R_{n, j} g_{i}\left[\mathbf{R}_{n}\right]\right)$, and then repeat this computation for each component. The difficulty in doing this is that $E_{i j}$ must obey two constraints: 1) $E_{i j}=E_{j i}$, and 2) for incompressible flow, $\nabla \cdot \mathbf{v}^{\text {amb }}=0$ and $\operatorname{tr} E=E_{i i}=0$. Thus there are only two independent components of $E$, and any physical $E$ can be built out of $E=\left(\begin{array}{cc}1 & 0 \\ 0 & -1\end{array}\right)$ and $E=\left(\begin{array}{ll}0 & 1 \\ 1 & 0\end{array}\right)$

Choosing $v^{\mathrm{amb}}=-E_{i j} r_{j}$ with

$$
E=\left(\begin{array}{cc}
1 & 0 \\
0 & -1
\end{array}\right)
$$

then shows $M_{i j x x}-M_{i j y y}=$ $\frac{1}{2} \sum_{n}\left(R_{n, i} g_{j}\left[\mathbf{R}_{n}\right]+R_{n, j} g_{i}\left[\mathbf{R}_{n}\right]\right)$, where the forces are the ones required to establish this particular flow. Only the combination $M_{i j x x}-M_{i j y y}$ can be identified. However, for any physical flow field $E^{\infty}, S_{h y}=M E^{\infty}$ will only depend on $M_{i j x x}$ and $M_{i j y y}$ in the combination $M_{i j x x}-M_{i j y y}$, because $E^{\infty}$ must have zero trace. We therefore choose to set $M_{i j x x}+M_{i j y y}=0$ to uniquely specify $M_{i j x x}$ and $M_{i j y y}$, though any arbitrary constant would yield the same physical results.

Similarly, $v^{\text {amb }}=-E_{i j} r_{j}$ with $E=\left(\begin{array}{ll}0 & 1 \\ 1 & 0\end{array}\right)$, yields $M_{i j x y}+M_{i j y x}=\frac{1}{2} \sum_{n}\left(R_{n, i} g_{j}\left[\mathbf{R}_{n}\right]+R_{n, j} g_{i}\left[\mathbf{R}_{n}\right]\right)-$ note again that $g_{j}$ are the forces for this flow. In this case, the combination $M_{i j x y}-M_{i j y x}$ is not determined, but is also not physically relevant. We arbitrarily choose the convention $M_{i j x y}=M_{i j y x}$.

\section{Computing effective viscosity with the grand resistance matrix}

Once the grand resistance matrix is known, it can be used to compute the effective viscosity. A particle with no external force or torque exerted on it, but subject to hydrodynamic perturbations from the surrounding fluid, will exert a force dipole $S_{i j}$ on the membrane. How does this depend on the external flow $\left(\mathbf{U}^{\infty}, \Omega^{\infty}, E_{i j}^{\infty}\right)$ ? The answer is hidden inside Eq. B2. In the absence of any external force or torque on the particle, Eq. B2 suggests

$$
\begin{aligned}
\left(\begin{array}{c}
\mathbf{F} \\
\tau
\end{array}\right)=\left(\begin{array}{c}
\mathbf{F}_{h y} \\
\tau_{h y}
\end{array}\right) & =\left(\begin{array}{l}
\mathbf{0} \\
0
\end{array}\right) \\
& =\left(\begin{array}{ccc}
A & \widetilde{B} & \widetilde{G} \\
B & C & \widetilde{H}
\end{array}\right)\left(\begin{array}{c}
\mathbf{U}^{\infty}-\mathbf{U} \\
\Omega^{\infty}-\Omega \\
E^{\infty}
\end{array}\right)
\end{aligned}
$$

with the solution

$$
\left(\begin{array}{c}
\mathbf{U}-\mathbf{U}^{\infty} \\
\Omega-\Omega^{\infty}
\end{array}\right)=\left(\begin{array}{cc}
A & \widetilde{B} \\
B & C
\end{array}\right)^{-1}\left(\begin{array}{c}
\widetilde{G} \\
\widetilde{H}
\end{array}\right) E^{\infty} .
$$

Upon substitution of this result into Eq. B2, an expression for the hydrodynamic dipole is obtained

$$
\begin{aligned}
S_{h y} & =-S \\
& =\left[M-(G H)\left(\begin{array}{cc}
A & \widetilde{B} \\
B & C
\end{array}\right)^{-1}\left(\begin{array}{c}
\widetilde{G} \\
\widetilde{H}
\end{array}\right)\right] E^{\infty}, \\
& \equiv \mathcal{M} E^{\infty}
\end{aligned}
$$

which is the quasi-2D analog to Eq. 5.21 of Ref. 36. The rank-4 tensor in square brackets in Eq. B7 is the sum of two terms. The first term $(M)$ indicates the dipolar response to attempted straining deformations by the fluid for a particle translating and rotating with the background flow. The second term corrects this response to impose conditions of vanishing external force/torque. By virtue of the symmetry relations on the resistance matrix (Eq. B3), this "correction term" is obtainable entirely from traditional RS calculations in the absence of $E^{\infty}$. M itself (up to the physically unimportant constants mentioned above) is obtainable from RS calculations for a static object $\mathbf{U}=\Omega=0$ with imposed $E^{\infty}$.

Appendix A shows that, if the force dipole obeys

$$
S_{i j} \approx-\alpha \eta_{m} A_{p}\left(\partial_{i} v_{j}^{\mathrm{amb}}+\partial_{j} v_{i}^{\mathrm{amb}}\right),
$$

then $\alpha$ is the intrinsic viscosity. This conclusion assumes slowly-varying external velocities. When flow is slowlyvarying enough to allow approximation as $\mathbf{v}^{\mathrm{amb}}(\mathbf{r}) \approx \mathbf{U}^{\infty}+$ $\Omega^{\infty} \times \mathbf{r}+E^{\infty} \cdot \mathbf{r}$, it is easily seen that Eq. B9 reduces to

$$
S_{i j} \approx-\alpha \eta_{m} A_{p}\left(E_{i j}^{\infty}+E_{j i}^{\infty}\right) .
$$


As noted previously, Eq. B9 holds only for isotropic particles; orientational averaging is required to yield this result in the general case.

$\alpha$ is calculated by picking a background flow field $E^{\infty}$, then computing $S_{i j}$ induced by the particle in that flow field directly from $\mathcal{R}$ by using Eq. B7 This is repeated for different particle orientations and values of the blob spacing and $\epsilon$, averaging over orientations, and extrapolating to zero spacing. $\alpha$ can be computed as

$$
\alpha=\frac{-\bar{S}_{x x}}{2 \eta_{m} A_{p}}
$$

for the choice $E^{\infty}=\left(\begin{array}{cc}1 & 0 \\ 0 & -1\end{array}\right)$.

In fact, because Eq. B7 determines the full tensor $\mathcal{M}$ that generates the hydrodynamic dipole response, orientational averaging may be carried out analytically, significantly improving computational speed. This resembles the approach of Ref. 55, but in two dimensions. When the particle is rotated, the tensor $\mathcal{M}_{i j k l}$ transforms to $R_{a i} R_{b j} R_{c k} R_{d l} \mathcal{M}_{i j k l}$, where $R_{a i}$ are the appropriate rotation matrices. The average over the angle of rotation follows as

$$
Z_{a b c d i j k l} \equiv \frac{1}{2 \pi} \int_{0}^{2 \pi} d \theta R_{a i} R_{b j} R_{c k} R_{d l} .
$$

These integrals are simple, if tedious, to evaluate - they are products of sines and cosines, which may be automated in Mathematica or constructed following Ref. 55. Then, the orientationally-averaged force dipole is given by

$$
\bar{S}_{a b}=-Z_{a b c d i j k l} \mathcal{M}_{i j k l} E_{c d}^{\infty}
$$

For present purposes, only $\bar{S}_{x x}$ is needed, which is given by

$$
\begin{aligned}
\bar{S}_{x x}=- & \frac{1}{4}\left(\mathcal{M}_{x x x x}-\mathcal{M}_{x x y y}+\mathcal{M}_{x y x y}+\mathcal{M}_{x y y x}\right. \\
& \left.+\mathcal{M}_{y x x y}+\mathcal{M}_{y x y x}-\mathcal{M}_{y y x x}+\mathcal{M}_{y y y y}\right)
\end{aligned}
$$

where $\mathcal{M}$ here is the tensor evaluated from Eq. B7 at a single orientation and we have assumed $E^{\infty}=\left(\begin{array}{cc}1 & 0 \\ 0 & -1\end{array}\right)$.

This approach has been used to reproduce the computations in Fig. 5. The maximum relative difference between the two approaches is less than $2 \times 10^{-5}$. Indeed, the two calculations should be completely equivalent, up to the differences between numerical versus analytical orientational averaging and the accuracy of the numerical method.

\section{REFERENCES}

${ }^{1}$ R. B. Gennis. Biomembranes: Molecular Structure and Function. Springer-Verlag, Berlin, 1989.

${ }^{2}$ M. J. Saxton and K. Jacobson. Single particle tracking: Applications to membrane dynamics. Annual Review of Biophysics and Biomolecular Structure, 26: 373-399, 1997.

${ }^{3}$ P G. Saffman and M. Delbrück. Brownian motion in biological membranes. Proc. Nat. Acad. Sci. USA, 72:3111, 1975.

${ }^{4}$ PG Saffman. Brownian motion in thin sheets of viscous fluid. Journal of Fluid Mechanics, 73(4):593, 1976.

${ }^{5}$ B D. Hughes, B A. Pailthorpe, and L R. White. The translational and rotational drag on a cylinder moving in a membrane. J. Fluid Mech., 110:349, 1981.

${ }^{6}$ Naomi Oppenheimer and Haim Diamant. Correlated diffusion of membrane proteins and their effect on membrane viscosity. Biophys. J., $96: 3041,2009$.

${ }^{7}$ Kerstin Weiß, Andreas Neef, Qui Van, Stefanie Kramer, Ingo Gregor, and Jörg Enderlein. Quantifying the diffusion of membrane proteins and peptides in black lipid membranes with 2-focus fluorescence correlation spectroscopy. Biophysical Journal, 105(2):455, 2013.

${ }^{8}$ S. Ramadurai, A. Holt, V. Krasnikov, G. Van Den Bogaart, J.A. Killian, and B. Poolman. Lateral diffusion of membrane proteins. J. Am. Chem. Soc., 131: $12650,2009$.

${ }^{9}$ Y. Gambin, R. Lopez-Esparza, M. Reffay, E. Sierecki, N. S. Gov, M.Genest, R. S. Hodes, and W. Urbach. Lateral mobility of proteins in liquid membranes revisited. Proc. Nat. Acad. Sci. USA, 103:2098, 2006.

${ }^{10}$ Brian A Camley, Michael G Lerner, Richard W Pastor, and Frank LH Brown. Strong influence of periodic boundary conditions on lateral diffusion in lipid bilayer membranes. The Journal of Chemical Physics, 143(24):243113, 2015.

${ }^{11}$ Richard M Venable, Helgi I Ingolfsson, Michael G Lerner, B Scott Perrin Jr, Brian A Camley, Siewert J Marrink, Frank LH Brown, and Richard W Pastor. Lipid and peptide diffusion in bilayers: The Saffman-Delbrück model and periodic boundary conditions. The Journal of Physical Chemistry B, 121(15): 3443-3457, 2017.

${ }^{12}$ Martin Vögele and Gerhard Hummer. Divergent diffusion coefficients in simulations of fluids and lipid membranes. The Journal of Physical Chemistry B, 120(33):8722-8732, 2016.

${ }^{13}$ Martin Vögele, Jürgen Köfinger, and Gerhard Hummer. Hydrodynamics of diffusion in lipid membrane simulations. Physical Review Letters, 120:268104, 2018.

${ }^{14}$ Andrew Zgorski and Edward Lyman. Toward hydrodynamics with solvent free lipid models: STRD martini. Biophysical Journal, 111(12):2689-2697, 2016.

${ }^{15}$ Alex J. Levine, T.B. Liverpool, and F.C. MacKintosh. Mobility of extended bodies in viscous films and membranes. Phys. Rev. E, 69:021503, 2004.

${ }^{16}$ Alex J. Levine, T.B. Liverpool, and F.C. MacKintosh. Dynamics of rigid and flexible extended bodies in viscous films and membranes. Phys. Rev. Lett., 93 : 038102, 2004.

${ }^{17}$ V. Prasad, S.A. Koehler, and E.R. Weeks. Two-particle microrheology of quasi-2d viscous systems. Phys. Rev. Lett., 97:176001, 2006.

${ }^{18}$ V Prasad and Eric R Weeks. Two-dimensional to three-dimensional transition in soap films demonstrated by microrheology. Physical Review Letters, 102 (17):178302, 2009.

${ }^{19}$ Christoph Klopp, Ralf Stannarius, and Alexey Eremin. Brownian dynamics of elongated particles in a quasi-two-dimensional isotropic liquid. Physical Review Fluids, 2(12):124202, 2017.

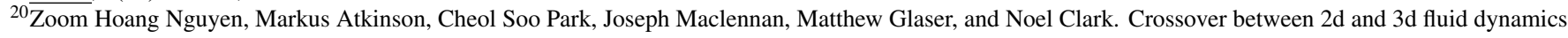
in the diffusion of islands in ultrathin freely suspended smectic films. Physical Review Letters, 105(26):268304, 2010. 
${ }^{21}$ Myung Han Lee, Clayton P Lapointe, Daniel H Reich, Kathleen J Stebe, and Robert L Leheny. Interfacial hydrodynamic drag on nanowires embedded in thin oil films and protein layers. Langmuir, 25(14):7976-7982, 2009.

${ }^{22}$ P. Cicuta, Sarah L. Keller, and Sarah L. Veatch. Diffusion of liquid domains in lipid bilayer membranes. J. Phys. Chem. B, 111:3328, 2007.

${ }^{23}$ Tristan T Hormel, Matthew A Reyer, and Raghuveer Parthasarathy. Two-point microrheology of phase-separated domains in lipid bilayers. Biophysical Journal, 109(4):732-736, 2015.

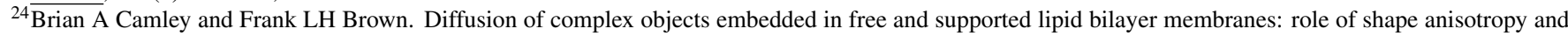
leaflet structure. Soft Matter, 9(19):4767, 2013.

${ }^{25}$ Ehsan Noruzifar, Brian A Camley, and Frank LH Brown. Calculating hydrodynamic interactions for membrane-embedded objects. The Journal of Chemical Physics, 141(12):124711, 2014

${ }^{26}$ M.L. Henle and A.J. Levine. Effective viscosity of a dilute suspension of membrane-bound inclusions. Physics of Fluids, 21:033106, 2009.

${ }^{27}$ D K. Lubensky and R E. Goldstein. Hydrodynamics of monolayer domains at the air-water interface. Phys. Fluids, 8:843, 1996.

${ }^{28}$ Alex J. Levine and F.C. MacKintosh. Dynamics of viscoelastic membranes. Phys. Rev. E, 66:061606, 2002.

${ }^{29}$ Ricardo Cortez, Lisa Fauci, and Alexei Medovikov. The method of regularized Stokeslets in three dimensions: Analysis, validation, and application to helical swimming. Phys. Fluids, 17:031504, 2005.

${ }^{30}$ Ricardo Cortez. The Method of Regularized Stokeslets. SIAM J. Sci. Comput., 23:1204, 2001.

${ }^{31} \mathrm{~V}$ Bloomfield, WO Dalton, and KE Van Holde. Frictional coefficients of multisubunit structures. I. Theory. Biopolymers: Original Research on Biomolecules, 5(2):135-148, 1967.

${ }^{32} \mathrm{~J}$ García de la Torre, $\mathrm{G}$ del Rio Echenique, and A Ortega. Improved calculation of rotational diffusion and intrinsic viscosity of bead models for macromolecules and nanoparticles. The Journal of Physical Chemistry B, 111(5):955-961, 2007.

${ }^{33}$ José García de la Torre, María L Huertas, and Beatriz Carrasco. Calculation of hydrodynamic properties of globular proteins from their atomic-level structure. Biophysical Journal, 78(2):719-730, 2000.

${ }^{34}$ Tatiana Kuriabova, Thomas R Powers, Zhiyuan Qi, Aaron Goldfain, Cheol Soo Park, Matthew A Glaser, Joseph E Maclennan, and Noel A Clark. Hydrodynamic interactions in freely suspended liquid crystal films. Physical Review E, 94(5):052701, 2016.

${ }^{35}$ Zhiyuan Qi, Zoom Hoang Nguyen, Cheol Soo Park, Matthew A Glaser, Joseph E Maclennan, Noel A Clark, Tatiana Kuriabova, and Thomas R Powers. Mutual diffusion of inclusions in freely suspended smectic liquid crystal films. Physical Review Letters, 113(12):128304, 2014.

${ }^{36}$ Sangtae Kim and Seppo J. Karrila. Microhydrodynamics: Principles and Selected Applications. Dover Publications, 2005.

${ }^{37}$ Brian A Camley and Frank LH Brown. Fluctuating hydrodynamics of multicomponent membranes with embedded proteins. The Journal of Chemical Physics, 141(7):075103, 2014.

${ }^{38}$ T.M Fischer. The drag on needles moving in a Langmuir monolayer. J. Fluid Mech., 498:123, 2004.

${ }^{39}$ B A. Camley, C. Esposito, T. Baumgart, and F L H. Brown. Lipid bilayer domain fluctuations as a probe of membrane viscosity. Biophys. J., 99:L44, 2010.

${ }^{40}$ Eugene P Petrov, Rafayel Petrosyan, and Petra Schwille. Translational and rotational diffusion of micrometer-sized solid domains in lipid membranes. Soft Matter, 8(29):7552, 2012.

${ }^{41}$ Eugene P. Petrov and Petra Schwille. Translational diffusion in lipid membranes beyond the Saffmann-Delbrück approximation. Biophys. J., 94:L41, 2008.

${ }^{42}$ Aurelia R Honerkamp-Smith, Francis G Woodhouse, Vasily Kantsler, and Raymond E Goldstein. Membrane viscosity determined from shear-driven flow in giant vesicles. Physical Review Letters, 111(3):038103, 2013.

${ }^{43}$ Myung Han Lee, Daniel H. Reich, Kathleen J. Stebe, and Robert L. Leheny. Combined passive and active microrheology study of protein-layer formation at an air-water interface. Langmuir, 26:2650, 2010.

${ }^{44}$ K.H. Kim, S.Q. Choi, J.A. Zasadzinski, and T.M. Squires. Interfacial microrheology of DPPC monolayers at the air-water interface. Soft Matter, 7(17): 7782-7789, 2011.

${ }^{45}$ Martin Vögele, Juergen Koefinger, and Gerhard Hummer. Finite-size corrected rotational diffusion coefficients of membrane proteins and carbon nanotubes from molecular dynamics simulations. The Journal of Physical Chemistry B, 2019.

${ }^{46}$ J. Domanski, S. J. Marrink, and L. V. Schafer. Transmembrane helices can induce domain formation in crowded model membranes. Biochimica et Biophysica Acta (BBA) - Biomembranes, 1818:984-994, 2012.

${ }^{47}$ M. Javanainen, H. Hammaren, L. Monticelli, J. H. Jeon, M. S. Miettinen, H. Martinez-Seara, R. Metzler, and L. Vattulainen. Anomalous and normal diffusion of proteins and lipids in crowded lipid membranes. Faraday Discuss., 161:397-417, 2013.

${ }^{48}$ J. E. Goose and M. S. P. Sansom. Reduced lateral mobility of lipids and proteins in crowded membranes. PLoS Comput. Biol., 161:e1003033, 2013.

${ }^{49}$ J. H. Jeon, M. Javanainen, H. Martinez-Sera, R. Metzler, and I. Vattulainen. Protein crowding in lipid bilayers gives rise to non-Gaussian anomalous lateral diffusion of phospholipids and proteins. Phys. Rev. X, 6:021006, 2016.

${ }^{50}$ Matti Javanainen, Hector Martinez-Seara, Ralf Metzler, and Ilpo Vattulainen. Diffusion of integral membrane proteins in protein-rich membranes. The Journal of Physical Chemistry Letters, 8(17):4308-4313, 2017.

${ }^{51} \mathrm{~B}$ Cichocki and BU Felderhof. Long-time self-diffusion coefficient and zero-frequency viscosity of dilute suspensions of spherical brownian particles. The Journal of Chemical Physics, 89(6):3705-3709, 1988.

52 Johan Bergenholtz and Norman J Wagner. The Huggins coefficient for the square-well colloidal fluid. Industrial \& Engineering Chemistry Research, 33(10): 2391-2397, 1994.

${ }^{53}$ Georges Bossis and John F Brady. The rheology of Brownian suspensions. The Journal of Chemical Physics, 91(3):1866-1874, 1989.

${ }^{54}$ Ronald G. Larson. The Structure and Rheology of Complex Fluids. Oxford University Press, 1999.

${ }^{55} \mathrm{DL}$ Andrews and T Thirunamachandran. On three-dimensional rotational averages. The Journal of Chemical Physics, 67(11):5026-5033, 1977. 